\title{
Ferroelectric PLZT Thick Films Grown by Poly(1-vinylpyrrolidone-co-vinyl acetate) (PVP/VA)-modified Sol-Gel Process
}

\author{
Zhongqiang $\mathrm{Hu}^{\mathrm{a}, *, 1}$, Beihai Ma ${ }^{\mathrm{a}}$, Meiya $\mathrm{Li}^{\mathrm{b}}$, Rachel E. Koritala ${ }^{\mathrm{a}}$, and Uthamalingam \\ Balachandran $^{\mathrm{a}}$ \\ a Energy Systems Division, Argonne National Laboratory, Argonne, IL 60439, USA \\ ${ }^{\mathrm{b}}$ School of Physics and Technology and Key Laboratory of Artificial Micro/Nano Structures of \\ Ministry of Education, Wuhan University, Wuhan 430072, China \\ * Corresponding author. Tel.: +1 630796 9139. E-mail address: z.hu@neu.edu (Z. Hu). \\ ${ }^{1}$ Current address: Department of Electrical and Computer Engineering, Northeastern \\ University, Boston, MA 02115, USA.
}




\section{Abstract}

We report the growth of ferroelectric $\mathrm{Pb}_{0.92} \mathrm{La}_{0.08} \mathrm{Zr}_{0.52} \mathrm{Ti}_{0.48} \mathrm{O}_{3}$ (PLZT) thick films using a poly(1-vinylpyrrolidone-co-vinyl acetate) (PVP/VA)-modified sol-gel process. A per-coating thickness of $\approx 0.66 \mu \mathrm{m}$ has been demonstrated using PVP/VA-modified solution, which is more than doubled that of the PLZT films grown by PVP-modified method, and nearly 6 times the percoating thickness of films prepared by conventional sol-gel process. PLZT thick films grown on LNO/Ni substrates exhibited denser microstructure, higher remanent polarization $\left(11 \mu \mathrm{C} / \mathrm{cm}^{2}\right)$ and dielectric tunability (45\%), lower leakage current density $\left(\approx 1.2 \times 10^{-8} \mathrm{~A} / \mathrm{cm}^{2}\right)$, and higher breakdown strength $(\approx 1.6 \mathrm{MV} / \mathrm{cm})$ than those for the samples grown on PtSi substrates. These results demonstrated great potential of using PVP/VA-modified sol-gel process for high power film capacitor applications.

Keywords: A. ceramics; B. sol-gel chemistry; C. X-ray diffraction; D. dielectric properties; D. ferroelectricity. 
Introduction

Ferroelectric thick films $(>1 \mu \mathrm{m})$ of lead zirconate titanate $(\mathrm{PZT})$ and lanthanum-doped PZT (PLZT) exhibit promising potentials for applications in actuators, sensors, DC/AC inverters, micro-electromechanical systems, and high power capacitors owing to their excellent dielectric, ferroelectric, and piezoelectric properties [1-7]. Sol-gel techniques have been used extensively for the fabrication of high quality ferroelectric films as they allow fast economical processing, easy stoichiometry control, and large coating area [8-10]. However, per-coating thickness that can be achieved by conventional 2-methoxyethanol sol-gel method is generally limited to $\approx 0.1 \mu \mathrm{m}[11$, 12]. To fabricate thick films, repeated coating and heating processes using low-viscosity solutions are necessary, because thicker single-coating films undergo cracking or delamination on gel-toceramic conversion; this is energy and time consuming, and thus unfavorable for industry production.

Several approaches have been proposed to achieve thick per-coating layer while avoiding crack or delamination in ferroelectric films. One is to use propanediol or acetic acid as solvents to increase the solubility and viscosity $[10,13,14]$. Tu et al. reported the use of propanediol to prepare high concentration PZT precursor solution $(1.6 \mathrm{M})$ for the fabrication of $1 \mu \mathrm{m}$ single-layer PZT films [13]. However, the high concentration diol solution is unstable in air and can lead to segregation after a few hours. Another approach is to use the composite sol-gel method by dispersing fine ceramic powders into the sol-gel precursor solution $[15,16]$; however, the ceramic powders resulted in considerable amount of micro-pores in the films and requires intricate 
filtrations steps to produce dense films [16]. The per-coating thickness could also be increased by adding organic polymers such as polyvinylpyrrolidone (PVP) or polyethylene glycol (PEG) into the sol-gel solutions [17-23]. The addition of PVP could help to achieve the desired viscosity for solutions and act as capping agent for metal precursors $[17,18]$. The higher viscosity of solutions leads to higher per-coating thickness. The strong hydrogen bonding between the $\mathrm{C}=\mathrm{O}$ groups of $\mathrm{PVP}$ and the $\mathrm{OH}$ groups of the metalloxane polymers prevents the films from cracking. By proper control and optimization of processing parameters such as sol concentration, spin coating parameters, heat treatment, and repeat times, thick films with desired thickness and minimum defects can be obtained [24]. In our earlier work, we reported the fabrication of high-quality PLZT films on platinized silicon (PtSi) and $\mathrm{LaNiO}_{3}$-buffred nickel (LNO/Ni) substrates by PVPmodified sol-gel deposition [25, 26]. However, the per-coating thickness appears to be limited to $\approx 0.27 \mu \mathrm{m}$ when PVP with an average molecular weight of $10000 \mathrm{~g} / \mathrm{mole}$ is used for making dense crack-free films [26].

In the present work, we report a modified sol-gel process to fabricate high-quality PLZT thick films by incorporating a new polymer poly(1-vinylpyrrolidone-co-vinyl acetate) (PVP/VA) into the precursor solution. To the authors' knowledge, this is by far the first report of using a PVP/AVmodified sol-gel process for making dense thick films. PVP/VA copolymer has been widely used as hot melt adhesives and photoresist binders [27]. The large molecular weight $(\approx 50000 \mathrm{~g} / \mathrm{mole})$ indicates that it will be effective in increasing the solution viscosity. More importantly, both PVP and VA in the monomer of PVP/VA exhibit $\mathrm{C}=\mathrm{O}$ groups. It could be expected that the strong 
hydrogen bonds between the amide groups of PVP/VA and the hydroxyl groups of the metalloxane polymers could impede the condensation reaction in PLZT films, promote the stress relaxation in the heating-up stage, and thus prevent the formation of cracks in thick films.

\section{Experimental}

High-purity nickel sheets polished by chemical mechanical planarization (MTI Corp., Richmond, CA) and platinized silicon wafers (Nova Electronic Materials, Flower Mound, TX) were used as substrates. Before being coated, the substrates were wipe-cleaned with acetone and methanol in sequence. Conductive oxide $\mathrm{LaNiO}_{3}$ (LNO) was first deposited on the nickel substrates by a sol-gel process to serve as a buffer layer [12, 28-29]. PLZT $(\mathrm{La} / \mathrm{Zr} / \mathrm{Ti}=8 / 52 / 48)$ stock solution of $0.5 \mathrm{M}$ concentration was prepared by a modified diol synthesis route by using following raw materials (all from Sigma-Aldrich): lead acetate trihydrate, lanthanum nitrate hexahydrate, zirconium propoxide, and titanium isopropoxide, as illustrated in Fig. 1. Lead acetate was first dissolved in 1,3-propanediol with a $\mathrm{Pb}$ : diol molar ratio of 1:5, and refluxed at $100{ }^{\circ} \mathrm{C}$ for $1.5 \mathrm{~h}$. A $20 \mathrm{~mol} \%$ excess of lead acetate was added to compensate for lead evaporation during the annealing. Zirconium propoxide, titanium isopropoxide, and lanthanum nitrate hexahydrate were then diluted in 2-methoxyethanol in sequence. The lead-diol solution was then added dropwise to the $\mathrm{Zr}$-Ti-La solution and refluxed at $115^{\circ} \mathrm{C}$ for $1 \mathrm{~h}$, and then cooled to room temperature. Finally, PVP/VA (with an average molecular weight of $50000 \mathrm{~g} / \mathrm{mole}$, and VP:VA $\approx 1.3: 1$ ) was added to the PLZT stock solution at a Pb:PVP/VA molar ratio of 1:1 (PVP/VA is defined by its monomer), 
and mixed for $1 \mathrm{~h}$ at room temperature. The PVP/VA-containing PLZT solution was aged for $>12$ $\mathrm{h}$ before spin coating. The aged PLZT solution was transparent and stable for $>3$ months.

After being filtered through a $0.2-\mu \mathrm{m}$ syringe filter (Whatman Inc., Sanford, ME), the PLZT solution was spin coated at $3000 \mathrm{rpm}$ for 30 s onto LNO/Ni and PtSi substrates, respectively. Pyrolysis of the green films was conducted via step-wise preheat treatment [25]. Films were dried at $200{ }^{\circ} \mathrm{C}$ for $5 \mathrm{~min}$, then preheated at $300^{\circ} \mathrm{C}$ for another $5 \mathrm{~min}$, and finally at $450{ }^{\circ} \mathrm{C}$ for $10 \mathrm{~min}$, by pushing the film into separate hot zones with designated temperatures in an electric furnace. After pyrolysis, the films were crystallized at $650{ }^{\circ} \mathrm{C}$ for $10 \mathrm{~min}$. The spin coating, pyrolysis, and crystallization steps were repeated to build up thick films with desired thickness. Final crystallization and densification were conducted at $650{ }^{\circ} \mathrm{C}$ for $30 \mathrm{~min}$. All the coating and heat treatments were performed in ambient atmosphere.

Microstructures of the PLZT thick films were observed with a field emission scanning electron microscope (SEM, Hitachi S4700). Phase identification was performed on a Bruker D8 AXS diffractometer with general area detector diffraction system. To measure the electrical properties, platinum top electrodes (diameter of $250 \mu \mathrm{m}$ and thickness of $100 \mathrm{~nm}$ ) were deposited by electron beam evaporation using a shadow mask. Polarization-field hysteresis loops were measured on a Radiant Technologies' Precision Premier II Tester. Dielectric measurements were made with an Agilent E4980A LCR meter using an oscillator level of $0.1 \mathrm{~V}$ in conjunction with a Signatone QuieTemp ${ }^{\circledR}$ probe station with hot stage. Current-voltage and the breakdown strength characteristics were measured by a Keithley 237 high-voltage source meter. 
Results and Discussion

Figure 2 shows $x$-ray diffraction patterns of the 2- $\mu$ m-thick PLZT films deposited on different substrates using PVP/VA-modified solutions. Well-crystallized, randomly oriented PLZT phases are observed in both samples without any traceable secondary phase. Pt top electrodes are also detected for the PLZT films grown on LNO/Ni substrates. The peaks of PLZT films deposited on PtSi (Fig. 2(b)) shifted to higher $2 \theta$ angle when compared to those grown on LNO/Ni substrates (Fig. 2(a)), which can be ascribed to the different strain states in the PLZT films on different substrates [30]. According to Bragg's equation $2 \mathrm{~d} \sin \theta=n \lambda$ ( $\lambda$ is the wavelength of $x$-ray), a peak shift to higher $2 \theta$ angle indicates a compression of d-spacing in the out-of-plane direction, which is a result of in-plane tensile strain in the films.

The x-ray diffraction $\sin ^{2} \psi$ method was used to determine the residual stresses $\sigma_{\phi}$ in the PLZT films, using the following equation [30]:

$$
\sigma_{\phi}=\left(\frac{E}{1+v}\right)_{(h k l)} \cdot \frac{1}{d_{\phi 0}} \cdot \frac{\partial d_{\phi \psi}}{\partial \sin ^{2} \psi},
$$

where $E$ and $v$ are Young's modulus $(72 \mathrm{GPa})$ and Poisson ratio $(0.3)$ of the PLZT films, $d_{\phi 0}$ and $d_{\phi \psi}$ are the lattice d-spacings measured in a stressed sample that is tilted at angles of zero and $\psi$, respectively. Keeping the rotation angle $\phi$ at $45^{\circ}$, the lattice d-spacings were measured as a function of $\sin ^{2} \psi$ for PLZT (211) and (220) diffraction peaks, as shown in Fig. 3. The negative and positive slopes determined from the linear fitting to the data indicate compressive stress in the PLZT grown on LNO-buffered nickel substrate, and tensile stress in the PLZT grown on PtSi substrate, respectively, which are in good agreement with those reported previously [20, 30]. The residual 
stresses were then determined by plugging the slopes into Eq. (1). A compressive stress of $\approx-110$ $\mathrm{MPa}$ and a tensile residual stress of $\approx 208 \mathrm{MPa}$ were calculated for the PLZT films grown on $\mathrm{LNO} / \mathrm{Ni}$ and PtSi substrates, respectively. Both values are respectively lower than the $\approx-370 \mathrm{MPa}$ and $\approx 250 \mathrm{MPa}$ reported for $\approx 2-\mu \mathrm{m}$-thick PLZT films deposited on similar substrates without PVP/VA addition [30]. Yao et al. also reported a reduced tensile stress in PZT films deposited on PtSi substrates when PEG was added to the precursor solution [20], which has been attributed to the looser morphology and lowered Young's modulus. Besides, the structure relaxation effect induced by the strong hydroxyl bonds between organic polymer and the gel network could also contribute to the reduced stresses observed in the PVP/VA-modified PLZT thick films [26].

Figure 4 shows the SEM images of the surface and cross-sectional morphology of the PLZT films deposited by PVP/VA-modified sol-gel process. The PLZT films grown on LNO/Ni substrates are dense and uniform, while the films on PtSi substrates are relatively porous. Porous structure is formed during the decomposition of the PLZT precursor and the PVP/VA in the pyrolysis process, since polymer and other organic compositions would produce a mixture of carbon oxides, water, nitrogen oxides, and other gases at high temperature. These gases would volatilize from inside of the films and leave behind many pores on the surface. During the later crystallization procedure, condensation reaction occurs with grain growth in the films and results in pore collapse and film densification. For the films on PtSi substrates which are suffered from tensile stress, the growth of the grain could be inhibited and thus not able to eliminate all the pores [17]. In contrast, the grain growth could be promoted under compressive stress, allowing dense 
and crack-free films to be formed [30]. This assumption is further supported by statistical calculations of the grain size using SEM images. The average grain sizes of the PLZT films on $\mathrm{PtSi}$ and LNO/Ni substrates are $179 \mathrm{~nm}$ and $224 \mathrm{~nm}$, respectively, indicating that tensile stress inhibits grain growth while compressive stress promotes grain growth. The PLZT films thickness after three deposition cycles are $\approx 2 \mu \mathrm{m}$ as determined from the cross-sectional image (inset of Fig.4(b)), corresponding to per-coating thickness of $\approx 0.66 \mu \mathrm{m}$, which is 6 times thicker than that measured for samples made by conventional 2-methoxyethanol sol-gel method (per-layer thickness $\approx 0.1 \mu \mathrm{m}$ ) and more than double that of the PVP-modified PLZT films (per-layer thickness $\approx 0.27 \mu \mathrm{m}$ ) [26]. The relatively high per-layer thickness should be attributed to the large molecular weight of PVP/VA used in the present study, as layer thickness is mainly determined by viscosity of the solution [25].

Figure 5 shows the polarization-field (P-E) hysteresis loops of the PLZT thick films grown on LNO/Ni and PtSi substrates. At a maximum electric field of $300 \mathrm{kV} / \mathrm{cm}$ and a frequency of 100 $\mathrm{Hz}$, we measured a remanent polarization $\left(\mathrm{P}_{\mathrm{r}}\right)$ of $\approx 11$ and $\approx 8 \mu \mathrm{C} / \mathrm{cm}^{2}$, and coercive electric field $\left(\mathrm{E}_{\mathrm{c}}\right)$ of $\approx 40$ and $\approx 35 \mathrm{kV} / \mathrm{cm}$, for PLZT films grown on LNO/Ni and PtSi substrates, respectively. This difference in P-E loops can be attributed to the different size and homogeneity of the structural grains, as well as the total strain in PLZT films. It has been reported that the in-plane compressive stress is beneficial to dipole alignment along the out-of-plane direction (i.e., parallel to the applied field) $[30,31]$. Therefore, the remanent polarization in PLZT films with compressive stress is higher than that from films with tensile stress. The remanent polarization of PVP/VA-modified 
PLZT films on LNO/Ni substrates is very close to that of PVP-modified PLZT films $\left(11.2 \mu \mathrm{C} / \mathrm{cm}^{2}\right)$ [26].

The dielectric constant and dielectric loss as a function of bias field are shown in Fig. 6. PLZT thick films grown on LNO/Ni and PtSi substrates display typical butterfly shaped hysteresis. At zero bias field, a dielectric constant of $\approx 740$ and dielectric loss of $\approx 0.07$ were observed for PLZT films on LNO/Ni substrates, and a dielectric constant of $\approx 780$ and dielectric loss of $\approx 0.06$ were measured for PLZT films grown on PtSi substrates, respectively. Under an applied field of 100 $\mathrm{kV} / \mathrm{cm}$, the dielectric tunabilities are $\approx 45 \%$ and $\approx 30 \%$ for PLZT films grown on LNO/Ni and PtSi substrates, respectively. The difference in tunability can also be attributed to the difference in residual stress for PLZT films grown on different substrates [30]. These values are comparable to those measured on PLZT films prepared by PVP-modified sol-gel process [26].

Time-relaxation data of leakage current density at $50 \mathrm{kV} / \mathrm{cm}$ are given in Fig. 7. The decay in dielectric relaxation current is fitted by Curie-von Schweidler equation [32]:

$$
J=J_{s}+J_{0} \cdot t^{-n}
$$

where $J_{s}$ is the steady-state current density, $J_{0}$ is a fitting constant, $t$ is the relaxation time in second, and $n$ is the slope of the $\log -\log$ plot. The calculated steady-state current densities are $\approx 1.2 \times 10^{-8}$ and $\approx 2.8 \times 10^{-8} \mathrm{~A} / \mathrm{cm}^{2}$ for the PLZT thick films grown on LNO/Ni and PtSi substrates, respectively. Higher leakage current density measured for the PLZT films grown on PtSi substrates is likely caused by the microstructure defects. These values are also comparable with that of PLZT films prepared by PVP-modified process and are reasonably low for capacitor applications $[25,26]$. 
Dielectric breakdown strength (BDS) was tested on PLZT samples in a top-to-bottom electrode configuration with a voltage increase of $5 \mathrm{~V}$ per second, and failures of the samples were defined by a 1-mA criterion. Weibull distribution was employed for failure behavior analysis [33,34]. Figure 8 shows the Weibull plot of breakdown field strength $\left(\mathrm{E}_{\mathrm{b}}\right)$ obtained from 20 measurements with PLZT films grown on on LNO/Ni and PtSi substrates. The mean breakdown strength values can be extracted from points where the fitting lines intersect with the horizontal line going through $\ln (\ln (1 /(1-\mathrm{p})))=0$. The sampling cumulative distribution parameter $\mathrm{p}$, using Bernard's approximation, is calculated by using $\mathrm{p}=(\mathrm{r}-0.3) /(\mathrm{N}+0.4)$ for each set of experimental data, where $\mathrm{N}=20$ is the total sample number, and $\mathrm{r}$ is the sample sequential number [34]. The data indicate that PLZT thick films deposited on LNO/Ni substrates exhibit higher mean breakdown strength $(\approx 1.6 \mathrm{MV} / \mathrm{cm})$ than that of films grown on PtSi substrates $(\approx 1.2 \mathrm{MV} / \mathrm{cm})$, which can be attributed to their dense microstructure, since porosity is detrimental to the breakdown strength [25]. Moreover, additional field strength that is required to compensate the residual compressive strain can also increase the breakdown strength for PLZT thick films grown on LNO/Ni substrates [30].

\section{Conclusions}

In summary, well-crystallized PLZT films were prepared by PVP/VA-modified sol-gel process on LNO/Ni and PtSi substrates. Residual stress analysis by XRD $\sin ^{2} \psi$ method revealed a compressive stress of $\approx-110 \mathrm{MPa}$ in the PLZT grown on LNO/Ni and a tensile stress of $\approx 208 \mathrm{MPa}$ 
in the PLZT on PtSi substrates. The film thickness after a single-layer deposition is $\approx 0.66 \mu \mathrm{m}$, which is 6 times of that measured for samples made by conventional 2-methoxyethanol sol-gel method and more than double that of the PVP-modified PLZT films. At room temperature, we measured a dielectric constant of $\approx 740$ and $\approx 780$ and dielectric loss of $\approx 0.07$ and $\approx 0.06$ for PLZT films grown on LNO/Ni and PtSi substrates, respectively. The PLZT thick films deposited on $\mathrm{LNO} / \mathrm{Ni}$ substrates show higher mean breakdown strength $(\approx 1.6 \mathrm{MV} / \mathrm{cm})$ than that grown on $\mathrm{PtSi}$ substrates $(\approx 1.2 \mathrm{MV} / \mathrm{cm})$. These results indicate that PLZT thick films prepared by PVP/VAmodified method exhibit comparable electrical properties to those grown by PVP-modified method. With much higher per-coating thickness, the PVP/VA-modified sol-gel process can be potentially useful for the fabrication of high power film capacitors.

\section{Acknowledgments}

This work was funded by the U.S. Department of Energy, Vehicle Technologies Program, under Contract DE-AC02-06CH11357. This study benefited from use of the Electron Microscopy Center (EMC) at Argonne National Laboratory.

\section{References}

[1] J.F. Scott, Applications of modern ferroelectrics, Science 315 (2007) 954-959.

[2] C.-B. Eom, S. Trolier-McKinstry, Thin-film piezoelectric MEMS, MRS Bulletin 37 (2012) 1007-1017. 
[3] U. Balachandran, D.K. Kwon, M. Narayanan, B. Ma, Development of PLZT dielectrics on base metal foils for embedded capacitors, Journal of the European Ceramic Society 30 (2010) 365368.

[4] I. Chilibon, J.N. Marat-Mendes, Ferroelectric ceramics by sol-gel methods and applications: a review, Journal of Sol-Gel Science and Technology 64 (2012) 571-611.

[5] Z. Hu, B. Ma, R.E. Koritala, U. Balachandran, Temperature-dependent energy storage properties of antiferroelectric $\mathrm{Pb}_{0.96} \mathrm{La}_{0.04} \mathrm{Zr}_{0.98} \mathrm{Ti}_{0.02} \mathrm{O}_{3}$ thin films, Applied Physics Letters 104 (26) (2014) 263902.

[6] Q. Zhou, S. Lau, D. Wu, K. Kirk Shung, Piezoelectric films for high frequency ultrasonic transducers in biomedical applications, Progress in Materials Science 56 (2011) 139-174.

[7] R. Dorey, R. Whatmore, Electroceramic thick film fabrication for MEMS, Journal of Electroceramics 12 (2004) 19-32.

[8] Z. Hu, B. Ma, S. Liu, M. Narayanan, U. Balachandran, Relaxor behavior and energy storage performance of ferroelectric PLZT thin films with different $\mathrm{Zr}$ /Ti ratios, Ceramics International 40 (1) (2014) 557-562.

[9] G.H. Haertling, Ferroelectric thin films for electronic applications, Journal of Vacuum Science \& Technology A: Vacuum, Surfaces, and Films 9 (1991) 414.

[10] A. Etin, G.E. Shter, G.S. Grader, G.M. Reisner, Interrelation of ferroelectricity, morphology, and thickness in sol-gel-derived $\mathrm{PbZr}_{\mathrm{x}} \mathrm{Ti}_{1-\mathrm{x}} \mathrm{O}_{3}$ films, Journal of the American Ceramic Society 90 (2007) 77-83. 
[11] K. Budd, S. Dey, D. Payne, Sol-gel processing of $\mathrm{PbTiO}_{3}, \mathrm{PbZrO}_{3}, \mathrm{~Pb}(\mathrm{ZrTi}) \mathrm{O}_{3}$ and $\mathrm{PbLa}(\mathrm{ZrTi}) \mathrm{O}_{3}$, British Ceramic Proceedings 36 (1985) 107-121.

[12] Z. Hu, B. Ma, S. Liu, M. Narayanan, U. Balachandran, Ceramic dielectric film capacitors fabricated on aluminum foils by chemical solution deposition, Materials Research Bulletin 52 (2014) 189-193.

[13] Y.L. Tu, M.L. Calzada, N.J. Phillips, S.J. Milne, Synthesis and electrical characterization of thin films of PT and PZT made from a diol-based sol-gel route, Journal of the American Ceramic Society 79 (1996) 441.

[14] B. Ma, S. Tong, M. Narayanan, S. Liu, S. Chao, U. Balachandran, Fabrication and dielectric property of ferroelectric PLZT films grown on metal foils, Materials Research Bulletin 64 (2011) 1124-1129.

[15] D.A. Barrow, T.E. Petroff, R.P. Tandon, M. Sayer, Characterization of thick lead zirconate titanate films fabricated using a new sol gel based process, Journal of Applied Physics 81 (1997) 876-881.

[16] D. Corker, Q. Zhang, R. Whatmore, C. Perrin, PZT ‘composite' ferroelectric thick films, Journal of the European Ceramic Society 22 (2002) 383-390.

[17] H. Kozuka, M. Kajimura, Single-step dip coating of crack-free $\mathrm{BaTiO}_{3}$ Films $>1 \mu$ m thick: effect of poly(vinylpyrrolidone) on critical thickness, Journal of the American Ceramic Society 83 (2000) 1056.

[18] S. Takenaka, H. Kozuka, Sol-gel preparation of single-layer, $0.75 \mu \mathrm{m}$ thick lead zirconate 
titanate films from lead nitrate-titanium and zirconium alkoxide solutions containing polyvinylpyrrolidone, Applied Physics Letters 79 (2001) 3485-3487.

[19] H. Kozuka, S. Takenaka, H. Tokita, M. Okubayashi, PVP-assisted sol-gel deposition of single layer ferroelectric thin films over submicron or micron in thickness, Journal of the European Ceramic Society 24 (2004) 1585-1588.

[20] K. Yao, S. Yu, F. Eng-Hock Tay, Residual stress analysis in ferroelectric $\mathrm{Pb}\left(\mathrm{Zr}_{0.52} \mathrm{Ti}_{0.48}\right) \mathrm{O}_{3}$ thin films fabricated by a sol-gel process, Applied Physics Letters 82 (2003) 4540.

[21] G.-T. Park, J.-J. Choi, C.-S. Park, J.-W. Lee, H.-E. Kim, Piezoelectric and ferroelectric properties of 1- $\mu \mathrm{m}$-thick lead zirconate titanate film fabricated by a double-spin-coating process, Applied Physics Letters 85 (2004) 2322.

[22] Z.H. Du, T.S. Zhang, J. Ma, Effect of polyvinylpyrrolidone on the formation of perovskite phase and rosette-like structure in sol-gel-derived PLZT films, Journal of Materials Research 22 (2007) 2195-2203.

[23] Y. Wang, X. Hao, J. Yang, J. Xu, D. Zhao, Fabrication and energy-storage performance of $(\mathrm{Pb}, \mathrm{La})(\mathrm{Zr}, \mathrm{Ti}) \mathrm{O}_{3}$ antiferroelectric thick films derived from polyvinylpyrrolidone-modified chemical solution, Journal of Applied Physics 112 (2012) 034105.

[24] J.-J. Choi, G.-T. Park, S.-M. Lee, H.-E. Kim, Sol-gel preparation of thick PZN-PZT film using a diol-based solution containing polyvinylpyrrolidone for piezoelectric applications, Journal of the American Ceramic Society 88 (2005) 3049-3054.

[25] S. Chao, B. Ma, S. Liu, M. Narayanan, U. Balachandran, Effects of pyrolysis conditions on 
dielectric properties of PLZT films derived from a polyvinylpyrrolidone-modified sol-gel process, Materials Research Bulletin 47 (2012) 907-911.

[26] B. Ma, S. Chao, M. Narayanan, S. Liu, S. Tong, R.E. Koritala, U. Balachandran, Dense PLZT films grown on nickel substrates by PVP-modified sol-gel method, Journal of Materials Science 48 (2012) 1180-1185.

[27] S.F. Mac Glee, U.S. Patent (2000) 6,077,898.

[28] J. Wu, J. Wang, Ferroelectric and impedance behavior of La- and Ti- codoped $\mathrm{BiFeO}_{3}$ thin films, Journal of the American Ceramic Society 93 (2010) 2795-2803.

[29] J. Wu, J. Wang, $\mathrm{BiFeO}_{3}$ thin films deposited on $\mathrm{LaNiO}_{3}$-buffered $\mathrm{SiO}_{2} / \mathrm{Si}$ Substrate, Journal of the American Ceramic Society 93 (2010) 1422-1426.

[30] B. Ma, S. Liu, S. Tong, M. Narayanan, U. Balachandran, Enhanced dielectric properties of $\mathrm{Pb}_{0.92} \mathrm{La}_{0.08} \mathrm{Zr}_{0.52} \mathrm{Ti}_{0.48} \mathrm{O}_{3}$ films with compressive stress, Journal of Applied Physics 112 (2012) 114117.

[31] J.-W. Lee, C.-S. Park, M. Kim, H.-E. Kim, Effects of residual stress on the electrical properties of PZT films, Journal of the American Ceramic Society 90 (2007) 1077-1080.

[32] K. Jonscher, Dielectric Relaxation in Solids, Chelsea Dielectrics Press, London, 1983.

[33] W. Weibull, A statistical distribution function of wide applicability, Journal of Applied Mechanics 18 (1951) 293-297.

[34] D.J. Smith, Reliability, Maintainability and Risk: Practical Methods for Engineers, Newnes, 2001. 


\section{Figure Captions}

Figure 1. Preparation of PLZT films via PVP/VA-modified sol-gel process.

Figure 2. XRD patterns of 2- $\mu$ m-thick PLZT films grown on (a) LNO/Ni and (b) PtSi substrates.

Figure 3. Lattice d-spacing as a function of $\sin 2 \psi$ measured for (a) PLZT (211) and (b) PLZT (220) diffraction peaks of PLZT thick films grown on LNO/Ni and PtSi substrates.

Figure 4. SEM images showing the surface morphology of PLZT films grown on (a) LNO/Ni and (b) PtSi substrates. The inset of Fig. 4(b) shows cross-sectional morphology of PLZT films on PtSi substrates.

Figure 5. P-E loops of PLZT thick films grown on LNO/Ni and PtSi substrates.

Figure 6. Field-dependent dielectric constant and loss of the PLZT thick films grown on LNO/Ni and PtSi substrates.

Figure 7. Current density relaxation measured at room temperature on PLZT thick films grown on LNO/Ni and PtSi substrates.

Figure 8. Weibull plot of dielectric breakdown strength for the PLZT films grown on LNO/Ni and PtSi substrates. 


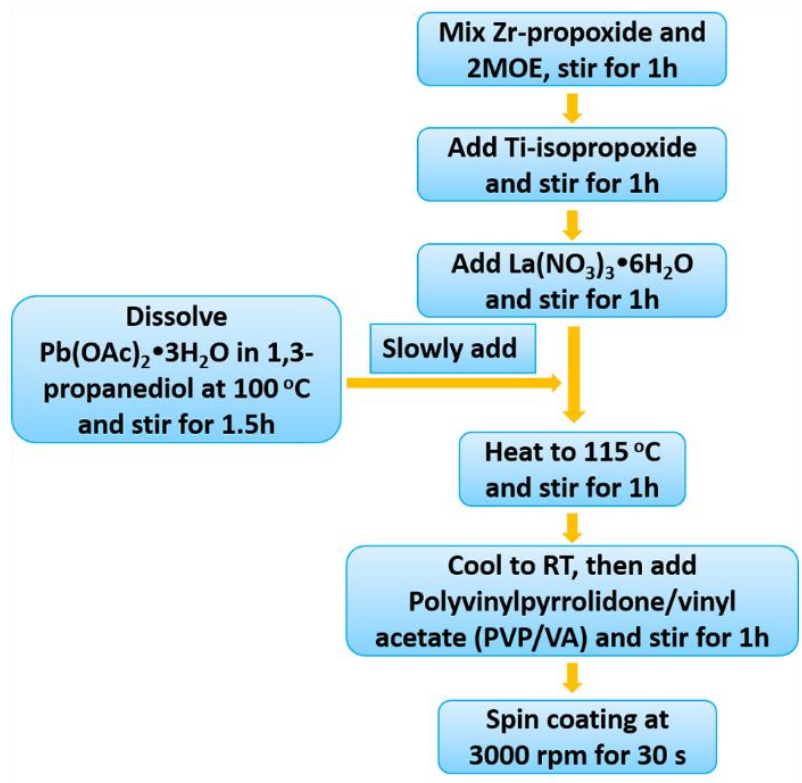




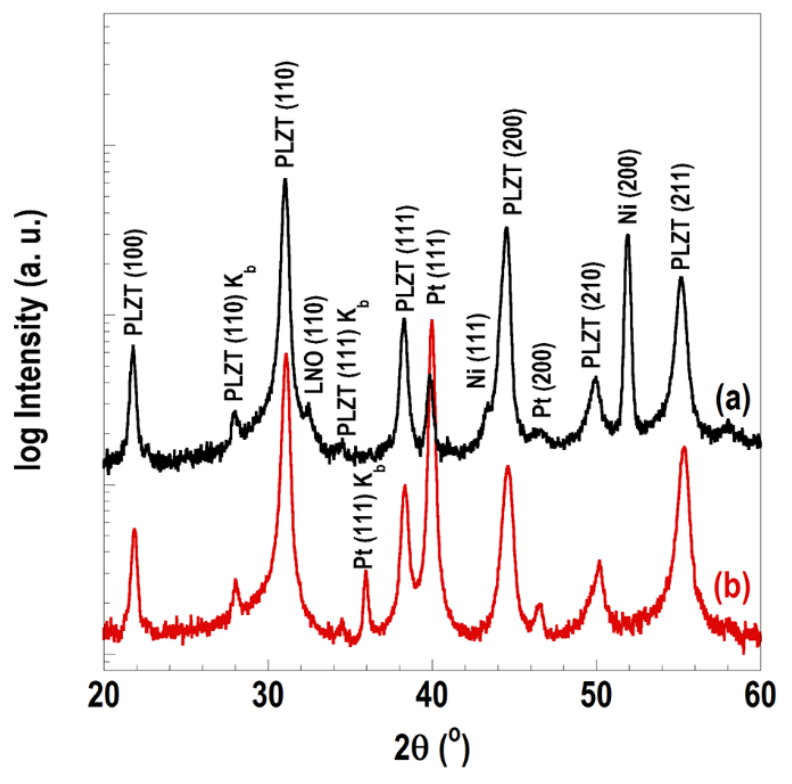



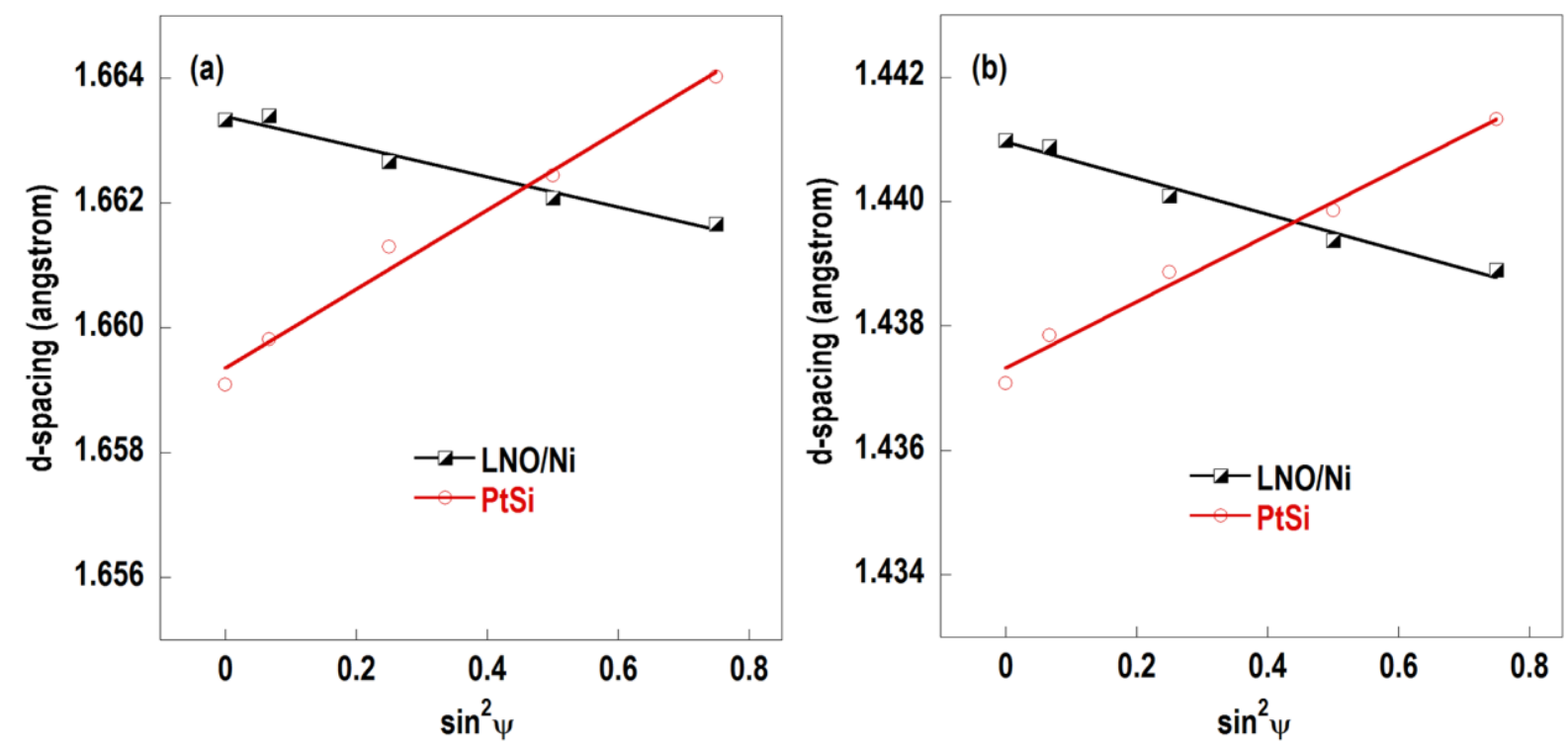

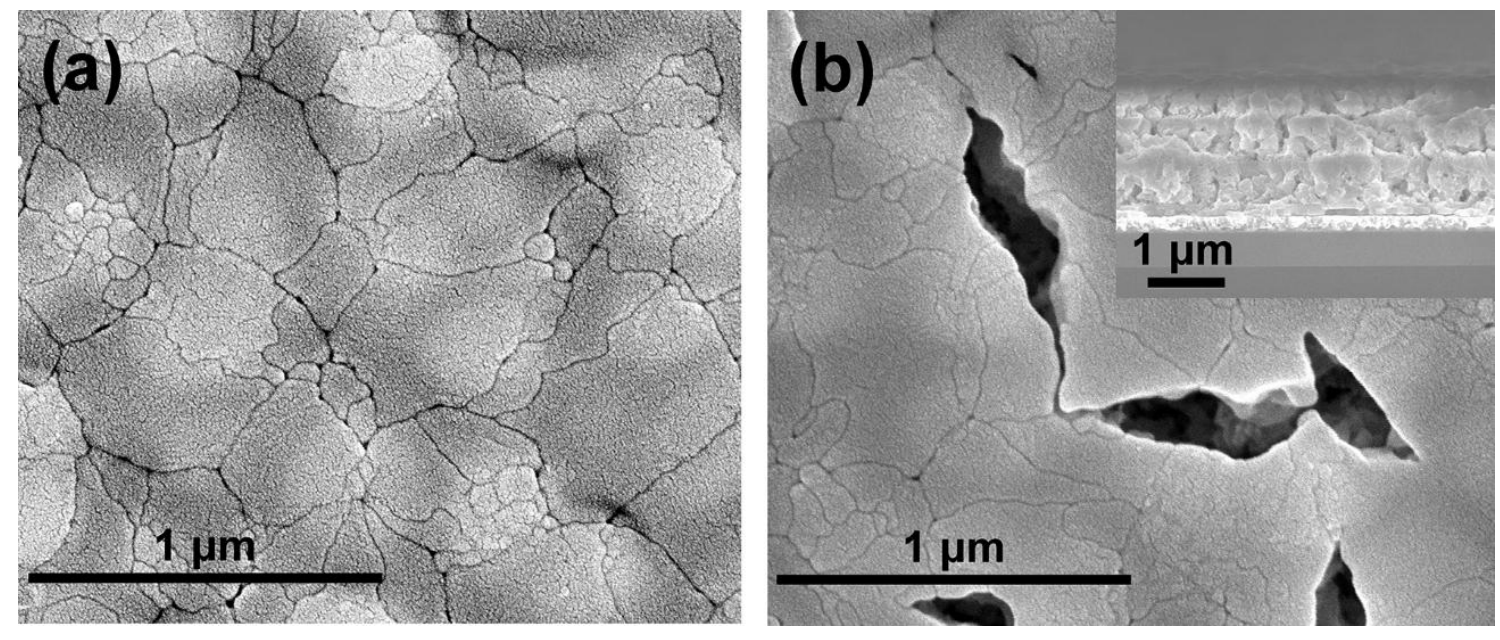


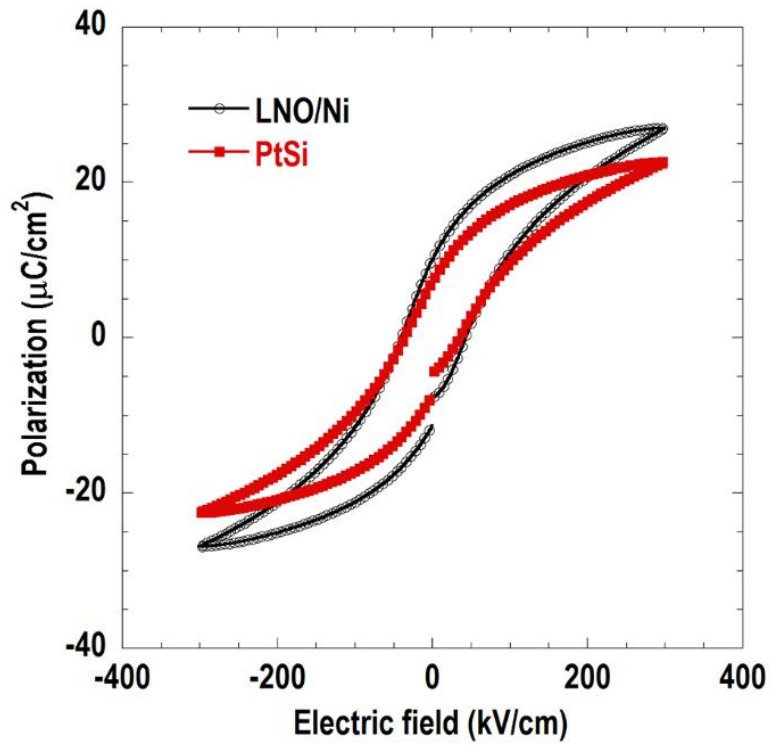




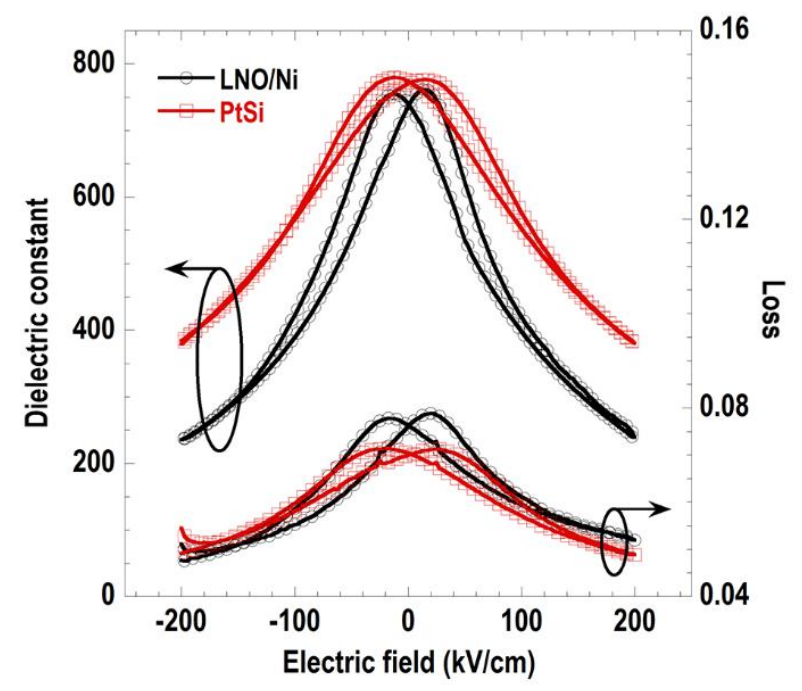




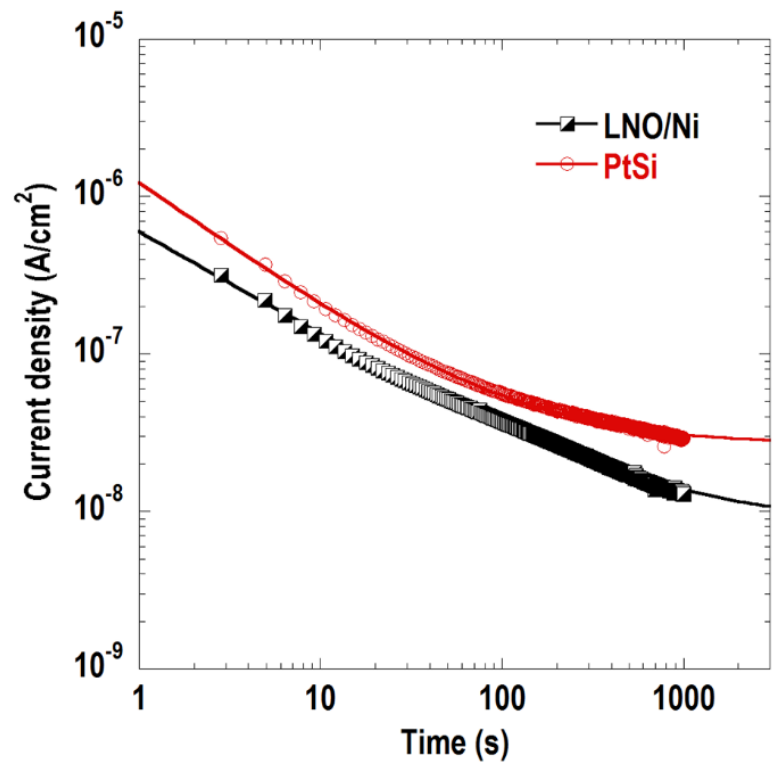




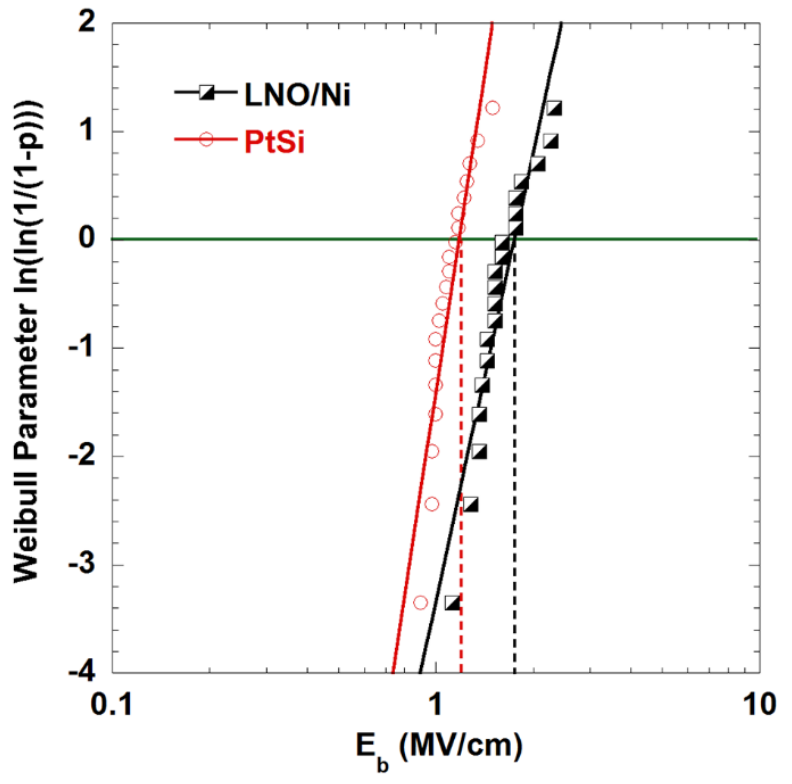




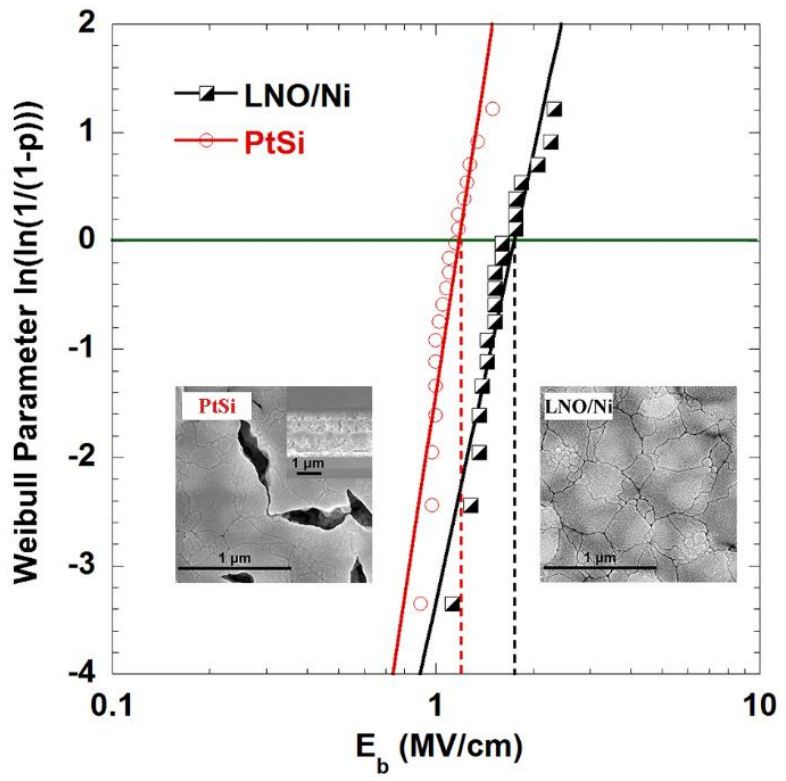

